\title{
Dexmedetomidine-induced neuroprotection: is it translational?
}

\author{
Yunzhen Wang ${ }^{1,2,}$ Ruquan $\mathrm{Han}^{2}$, and Zhiyi Zuo ${ }^{1}$ \\ ${ }^{1}$ Department of Anesthesiology, University of Virginia, Charlottesville, Virginia 22901, USA \\ ${ }^{2}$ Department of Anesthesiology, Beijing Tian Tan Hospital, Capital Medical University, Beijing 100050, China
}

\begin{abstract}
Dexmedetomidine is often used in anesthesia and critical care medicine practice to sedate patients. Its neuroprotective effects have been shown in various ischemic and hemorrhagic brain injury models of animals. Randomized clinical trials have indicated that dexmedetomidine can improve outcome of patients under intensive care. Clinical trials are needed to determine whether dexmedetomidine can provide neuroprotection against ischemic and hemorrhagic stroke.
\end{abstract}

Key words:Dexmedetomidine; ischemic stroke; neuroprotection; ICU

Dexmedetomidine has become a commonly used drug in anesthesia and critical care medicine practice. The molar mass of dexmedetomidine is $200.28 \mathrm{~g} / \mathrm{mol}$ with formula of $\mathrm{C}_{13} \mathrm{H}_{16} \mathrm{~N}_{2}$. The generic name of dexmedetomidine is precedex or dexdor. It is a selective agonist of $\alpha_{2}$-adrenergic receptor with anxiolytic, sedative and analgesic effects. It provides sedation without inhibiting respiration. Patient can be cooperative or semi-arousal under the sedation with dexmedetomidine. Its main side-effects include bradycardia and hypotension, which can be treated pharmacologically. In this review, we will discuss the potential neuroprotective effects of dexmedetomidine.

\section{Evidences from animal models}

It has been reported that dexmedetomidine has neuroprotective effects in animals with various insults to the brain. Dexmedetomidine attenuates central sympathetic activity and decreases anesthetic requirements. Hoffman et al. deter- mined the effects of dexmedetomidine on neurological and histopathological outcome after incomplete cerebral ischemia in rats about 25 years ago [1]. The results show that dexmedetomidine given during brain ischemia improved neurological and histopathological outcome after incomplete ischemia in rats anesthetized with fentanyl and nitrous oxide. This effect was reversed by atipamezole, indicating that the effect is mediated by $\alpha_{2}$-adrenergic receptors. Improvement with dexmedetomidine was not mediated by changes in plasma glucose or other physiologic variables during ischemia. It was concluded that dexmedetomidine decreased ischemic brain injury after incomplete cerebral ischemia by decreasing sympathetic activity. In another study with using a model of focal cerebral ischemia caused by occluding the left internal carotid, anterior and middle cerebral arteries for $2 \mathrm{~h}$ [2], rabbits received dexmedetomidine or normal saline $10 \mathrm{~min}$ after the occlusion. A plasma level of dexmedetomidine was maintained at $4.0 \pm 0.15 \mathrm{ng} /$ $\mathrm{ml}$. The area of ischemic neuronal injury in the cortex was significantly decreased by about $47.6 \%$ in the group treated with dexmedetomidine. These results suggest that treatment with dexmedetomidine, at a dose to reduce the requirements of anesthetics by $50 \%$, provides neuroprotection against focal cerebral ischemia.

Perinatal asphyxia can lead to death and severe disability. Brain hypoxia-ischemia (HI) injury is the major pathophysiology contributing to death and severe disability after perinatal asphyxia. Ren et al. studied the effects of dexmedetomidine on seven-day old Sprague Dawley rats with left 
brain HI [3]. These animals received intraperitoneal dexmedetomidine various times after brain HI. They were evaluated 7, 28 and 43 days after the brain HI. Dexmedetomidine induced neuroprotective effects when it was applied within $1 \mathrm{~h}$ after the brain HI. The brain cell and tissue loss as well as neurological and cognitive dysfunction evaluated from 28 days after brain HI were attenuated by dexmedetomidine. These results indicate that dexmedetomidine post-treatment induces neuroprotection against HI-induced brain injury in the neonatal rats. This protective effect may be mediated by inhibiting inflammation in the ischemic brain tissues via $\alpha_{2}-$ adrenergic receptor activation.

In addition to the protective effects against ischemia, dexmedetomidine also shows neuroprotection in rats with subarachnoid hemorrhage (SAH) [4]. Dexmedetomidine was applied in two-month old Sprague Dawley rats with SAH. The SAH model was established by perforating the junction of the right middle and anterior cerebral arteries. Animals received dexmedetomidine immediately or $2 \mathrm{~h}$ after SAH. They were evaluated 2 days after SAH. SAH worsened neurological functions, induced brain edema and increased blood-brain barrier permeability. These detrimental effects were reduced by dexmedetomidine post-treatment. However, the neuroprotective effects of dexmedetomidine was abolished by inhibition of extracellular signal-regulated kinase (ERK) activation. The conclusion in this study is that dexmedetomidine post-treatment induces neuroprotection to reduce SAH-induced brain injury. This effect may be mediated by activating ERK.

\section{Evidence from human studies}

Dexmedetomidine also shows some neuroprotective effects in human. Medications inducing sedation and analgesia are often administrated to patients ventilated mechanically to reduce anxiety and pain and to permit invasive procedures in the intensive care unit (ICU). The MENDS (maximizing efficacy of targeted sedation and reducing neurological dysfunction) randomized controlled trial was designed to determine whether dexmedetomidine reduces the duration of delirium and coma in mechanically ventilated ICU patients while providing adequate sedation as compared with lorazepam [5]. Total 106 adult medical and surgical ICU patients ventilated mechanically in 2 tertiary care centers were randomly assigned to two groups. They were sedated by dexmedetomidine or lorazepam for as long as 120 hours. Patients were assessed for delirium with using the Confusion Assessment Method for ICU patients twice daily. Patients sedated with dexmedetomidine had more coma-free or coma and delirium-free days and a lower prevalence of coma than sedation with lorazepam. More patients sedated with dexmedetomidine completed post-ICU neuropsychological testing and had similar scores in those tests assessing global cognitive, motor speed, and attention functions. In those ICU patients that were managed with individualized targeted sedation, dexmedetomidine infusion led to more coma- or coma and delirium-free days than with a lorazepam infusion at the targeted level of sedation.

Interestingly, it has been shown that benzodiazepines and $\alpha_{2}$-adrenoceptor agonists the effects of benzodiazepines and $\alpha_{2}$-adrenoceptor agonists on innate immunity and mortality in animals with infection may be different. Benzodiazepines impair neutrophil and macrophage functions $[6,7]$. $\alpha_{2}$-adrenoceptor agonists increase macrophage phagocytosis but have limited effects on neutrophil functions $[8,9]$. Benzodiazepines increase the mortality of animals with infection [10] while $\alpha_{2}$-adrenoceptor agonists improve mortality in animals with infection [11]. In a sub-analysis of the MENDS data, the authors compared the effect of dexmedetomidine with that of lorazepam on septic patients [12]. Among them, 63 patients had sepsis and 40 patients were without sepsis. Septic patients sedated with lorazapam had 3.2 more days with delirium/coma on average, 1.5 more days with delirium and more days requiring a ventilator. These protective effects of dexmedetomidine were enhanced in septic patients than patients without sepsis. Mortality at 28 days were reduced by $70 \%$ in septic patients sedated by dexmedetomidine as compared to the patients in the lorazepam group. A randomized, double-blinded, placebo-controlled trial was just published Lancet. The study determined the effectiveness of dexmedetomidine to prevent delirium in elderly patients after non-cardiac surgery [13]. Total 700 patients aged 


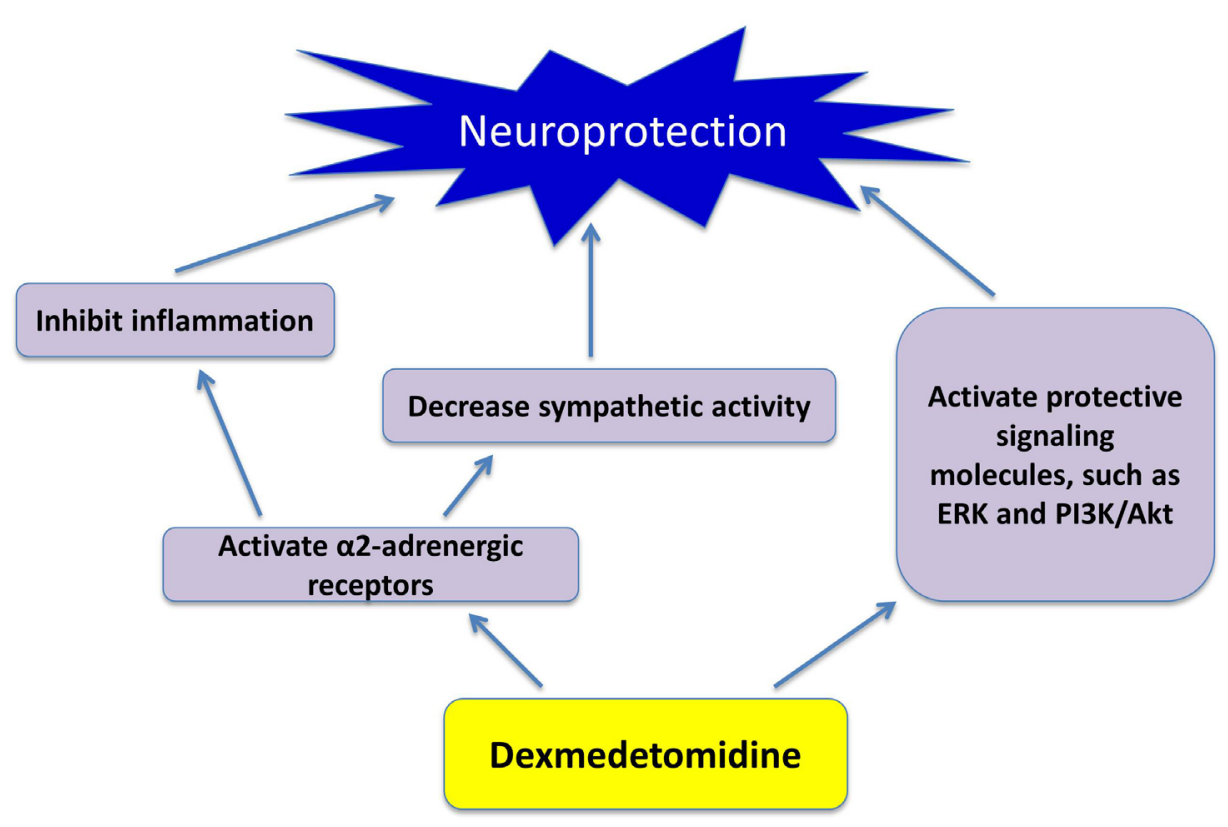

Figure 1. Potential mechanisms for dexmedetomidine to induce neuroprotection (ERK: extracellular signal-regulated kinase; PI3K: phosphatidylinositol 3-kinase)

65 years or older were assigned to receive either placebo or dexmedetomidine $(0.1 \mu \mathrm{g} / \mathrm{kg} / \mathrm{h})$. The incidence of postoperative delirium was significantly lower in the dexmedetomidine group than in the placebo group. Regarding the safety, the incidence of hypertension and tachycardia were higher with placebo than with dexmedetomidine. Thus, it was concluded that a low-dose of dexmedetomidine significantly decreased the occurrence of delirium during the first 7 days after non-cardiac surgery in elderly ICU patients.

\section{Potential mechanisms}

As described above, dexmedetomidine can inhibit inflammation and decrease sympathetic activity via $\alpha_{2}$-adrenergic receptor activation. It also can activate some protective signal pathway, such as ERK and phosphoinositide 3-kinase (PI3K) /Akt pathway, to provide neuroprotection [14]. The activation of ERK by dexmedetomidine may be via mechanisms independent of $\alpha_{2}$-adrenergic receptor activation [15] (Figure 1).

In summary, dexmedetomidine can provide neuroprotection in animals with various insults to the brain. It appears that dexmedetomidine also improves neurological outcome in patients without primary neurological diseases. It improved neurological dysfunction, such as delirium, in me- chanically ventilated patients and in elderly ICU patients after non-cardiac surgery. It also decreased mortality in septic patients. It is not known yet whether dexmedetomidine can provide neuroprotection against ischemia or hemorrhagic brain injury in human. Prospective clinical studies are needed to determine whether those effects shown in animals are translational in human.

\section{Disclosure of Funding}

Research in Dr. Zuo's laboratory is currently supported by grants (GM098308 and AG047472) from the National Institutes of Health, Bethesda, MD, and the Robert M. Epstein Professorship endowment, University of Virginia, Charlottesville, VA.

Conflict Interests Disclosure: The authors have no conflicting interests to disclose.

Corresponding Authors: Zhiyi Zuo, MD, PhD Robert M. Epstein Professor of Anesthesiology, Professor of Neurological Surgery, and Neuroscience, University of Virginia. zz3c@virginia.edu; or Ruquan Han, MD, PhD, Professor and Chair, Department of Anesthesiology, Beijing Titan Hospital, Capital University. Tele: 86-10-67096660, Fax: 86-1067031947,e-mail: ruquan.han@yahoo.com 
Editor: Renyu Liu, MD; PhD. Associate Professor, Director of Preoperative Medicine, Department of Anesthesiology and Critical Care; Perelman School of Medicine at the University of Pennsylvania, 336 John Morgan building, 3620 Hamilton Walk, Philadelphia, PA 19104 . Phone: 2157461485 ; FAX: 2153495078. liur@uphs.upenn.edu

\section{Additional publication details}

Journal short name: Transl Perioper \& Pain Med

Received Date: September 16, 2015

Accepted Date: Nov 11, 2016

Published Date: Nov 12, 2016

Transl Perioper \& Pain Med 2016; 1(4):15-19

\section{Citation and Copyright}

Citation: Wang Y, Han R, Zuo Z. Dexmedetomidineinduced neuroprotection: is it translational? Transl Perioper \& Pain Med 2016; 1(4): 15-19

Copyright: () 2016 Wang Y., et al. This is an open-access article distributed under the terms of the Creative Commons Attribution License, which permits unrestricted use, distribution, and reproduction in any medium, provided the original author and source are credited.

\section{References}

1. Hoffman WE, Kochs E, Werner C, Thomas C, Albrecht RF. Dexmedetomidine improves neurologic outcome from incomplete ischemia in the rat. Anesthesiology. 1991;75:328-32.

2. Maier C, Steinberg GK, Sun GH, Zhi GT, Maze M. Neuroprotection by the alpha 2-adrenoreceptor agonist dexmedetomidine in a focal model of cerebral ischemia. Anesthesiology. 1993;79(2):306-12. PubMed PMID: 8102042.

3. Ren X, Ma H, Zuo Z. Dexmedetomidine Postconditioning Reduces Brain Injury after Brain Hypoxia-Ischemia in Neonatal Rats. Journal of neuroimmune pharmacology : the official journal of the Society on NeuroImmune
Pharmacology. 2016;11(2):238-47. doi: 10.1007/s11481016-9658-9. PubMed PMID: 26932203.

4. Wang Y, Han R, Zuo Z. Dexmedetomidine post-treatment induces neuroprotection via activation of extracellular signal-regulated kinase in rats with subarachnoid haemorrhage. British journal of anaesthesia. 2016;116(3):384-92. doi: 10.1093/bja/aev549. PubMed PMID: 26865131; PubMed Central PMCID: PMC4748945.

5. Pandharipande PP, Pun BT, Herr DL, Maze M, Girard TD, Miller RR, et al. Effect of sedation with dexmedetomidine vs lorazepam on acute brain dysfunction in mechanically ventilated patients: the MENDS randomized controlled trial. Jama. 2007;298(22):2644-53. doi: 10.1001/ jama.298.22.2644. PubMed PMID: 18073360.

6. Finnerty M, Marczynski TJ, Amirault HJ, Urbancic M, Andersen BR. Benzodiazepines inhibit neutrophil chemotaxis and superoxide production in a stimulus dependent manner; PK-11195 antagonizes these effects. Immunopharmacology. 1991;22(3):185-93. PubMed PMID: 1663497.

\section{Kim SN, Son SC, Lee SM, Kim CS, Yoo DG, Lee}

SK, et al. Midazolam inhibits proinflammatory mediators in the lipopolysaccharide-activated macrophage. Anesthesiology. 2006;105(1):105-10. PubMed PMID: 16810001.

8. Miles BA, Lafuse WP, Zwilling BS. Binding of alpha-adrenergic receptors stimulates the anti-mycobacterial activity of murine peritoneal macrophages. Journal of neuroimmunology. 1996;71(1-2):19-24. PubMed PMID: 8982098.

9. Nishina K, Akamatsu H, Mikawa K, Shiga M, Maekawa N, Obara H, et al. The effects of clonidine and dexmedetomidine on human neutrophil functions. Anesthesia and analgesia. 1999;88(2):452-8. PubMed PMID: 9972773.

10. Domingues-Junior M, Pinheiro SR, Guerra JL, Palermo-Neto J. Effects of treatment with amphetamine and diazepam on Mycobacterium bovis-induced infection in hamsters. Immunopharmacology and immunotoxicolo- 
gy. 2000;22(3):555-74. doi: 10.3109/08923970009026012.

PubMed PMID: 10946832.

11. Hofer S, Steppan J, Wagner T, Funke B, Lichten-

stern C, Martin E, et al. Central sympatholytics prolong survival in experimental sepsis. Critical care. 2009;13(1):R11. doi: 10.1186/cc7709. PubMed PMID: 19196475; PubMed Central PMCID: PMC2688128.

12. Pandharipande PP, Sanders RD, Girard TD, McGrane S, Thompson JL, Shintani AK, et al. Effect of dexmedetomidine versus lorazepam on outcome in patients with sepsis: an a priori-designed analysis of the MENDS randomized controlled trial. Critical care. 2010;14(2):R38. doi: 10.1186/cc8916. PubMed PMID: 20233428; PubMed Central PMCID: PMC2887145.

\section{Su X, Meng ZT, Wu XH, Cui F, Li HL, Wang} DX, et al. Dexmedetomidine for prevention of delirium in elderly patients after non-cardiac surgery: a randomised, double-blind, placebo-controlled trial. Lancet. 2016. doi: 10.1016/S0140-6736(16)30580-3. PubMed PMID: 27542303.

14. Zhu YM, Wang CC, Chen L, Qian LB, Ma LL, Yu J, Zhu MH, Wen CY, Yu LN and Yan M. Both PI3K/Akt and ERK1/2 pathways participate in the protection by dexmedetomidine against transient focal cerebral ischemia/reperfusion injury in rats. Brain Res, 2013; 1494:1-8 PubMed PMID:23219579

15.Dahmani S, Paris A, Jannier V, Hein L, Rouelle D, Scholz J, Gressens P and Mantz J. Dexmedetomidine increases hippocampal phosphorylated extracellular signal-regulated protein kinase 1 and 2 content by an alpha 2-adrenoceptor-independent mechanism: evidence for the involvement of imidazoline I1 receptors. Anesthesiology, 2008; 108 (3):457-466 PubMed PMID:18292683 